\title{
Adaptive spatial-temporal filtering applied to x-ray fluoroscopy angiography
}

\author{
Gert Schoonenberg $^{\mathrm{a}, \mathrm{b}}$, Marc Schrijver ${ }^{\mathrm{c}}$, Qi Duan ${ }^{\mathrm{b}}$, Richard Kemkers ${ }^{\mathrm{d}}$, Andrew Laine ${ }^{\mathrm{b}}$ \\ ${ }^{a}$ Dept. of Biomedical Engineering, Eindhoven Univ. of Technology, Postbus 513, $5600 \mathrm{MB}$ \\ Eindhoven, The Netherlands; \\ ${ }^{\mathrm{b}}$ Dept. of Biomedical Engineering, Columbia Univ., 351 Engineering Terrace - MC 8904, 1210 \\ Amsterdam Ave., New York, NY 10027 USA; \\ ${ }^{\mathrm{c}}$ Philips Medical Systems, P.O. Box 10.000, 5680 DA Best, The Netherlands; \\ ${ }^{d}$ Philips Medical Systems North America, 22100 Bothell-Everett Hwy (mailstop 713), Bothell, WA \\ 98021 USA
}

\begin{abstract}
Adaptive filtering of temporally varying X-ray image sequences acquired during endovascular interventions can improve the visual tracking of catheters by radiologists. Existing techniques blur the important parts of image sequences, such as catheter tips, anatomical structures and organs; and they may introduce trailing artifacts. To address this concern, an adaptive filtering process is presented to apply temporal filtering in regions without motion and spatial filtering in regions with motion. The adaptive filtering process is a multi-step procedure. First a normalized motion mask that describes the differences between two successive frames is generated. Secondly each frame is spatially filtered using the specific motion mask to specify different types of filtering in each region. Third an IIR filter is then used to combine the spatially filtered image with the previous output image; the motion mask thus serves as a weighted input mask to determine how much spatial and temporal filtering should be applied. This method results in improving both the stationary and moving fields. The visibility of static anatomical structures and organs increases, while the motion of the catheter tip and motion of anatomical structures and organs remain unblurred and visible during interventional procedures.
\end{abstract}

Keywords: Adaptive filtering, spatial filtering, temporal filtering, angiography, interventional radiology, minimally invasive surgery, catheter tracking, guide-wire tracking, image enhancement

\section{INTRODUCTION}

Interventional procedures are becoming more frequent and preferred due to the negative side effects of conventional surgery protocols, including the risk of trauma, infection and patient recovery time secondary to large incisions. During the procedure radiologists use temporally varying x-ray image sequences, or x-ray fluoroscopy angiography, to navigate the catheter through the vasculature from the groin to the heart. However, during such procedures, hundreds of x-ray images are acquired, with possible risk to the patients' and the doctors' health due to the exposure of radiation. Thus, in clinical applications, low-dose $\mathrm{x}$-ray fluoroscopy is preferred to reduce such hazard. However this often results in degrading the image quality by reducing the signal-to-noise ratio. A typical x-ray fluoroscopic angiogram is shown in Fig 1 (b). The catheter part is indicated with black arrows. The white arrow indicates the point where the guide-wire ends in the catheter. The contrast of only the catheter is very low. As a result, denoising and enhancing $\mathrm{x}$-ray fluoroscopic images have become a focus of interest in low-dose x-ray fluoroscopic imaging.

Generally, denoising methods can be categorized into two major classes based on the information used: spatial filtering and temporal filtering. Spatial filtering treats frames separately and utilizes 2-D imaging denoising techniques to improve image quality frame by frame. Spatial filtering methods contain traditional linear filtering ${ }^{1}$, median filtering ${ }^{2}$, linear/median hybrid filtering ${ }^{3}$, edge-preserving filtering ${ }^{4,5}$, or probability based methods, or other image enhancement methods $^{6-9}$. In order to improve the performance of noise-suppression, some of the spatial filtering methods take the advantage of wavelet expansion and multi-resolution frameworks $s^{3,10,11}$ to efficiently separate signal from noise. Temporal filtering methods have also been investigated actively in last decade. Many methods have been applied, 
including traditional FIR/IIR filtering, probability based methods ${ }^{12}$ like Bayesian analyses ${ }^{13}$ and maximum likelihood ${ }^{14}$, $\operatorname{etc}^{12,15}$. One advantage of temporal filtering over spatial filtering is that without averaging between neighboring pixels in the stationary areas, temporal filtering can preserve the fine anatomical structure while suppressing noise. Whereas for most spatial filtering methods, there is a trade-off between feature preserving and denoising. However, by averaging across the time dimension, temporal filtering can potentially introduce trailing artifacts in the moving regions, which is made worse by the fact that the catheter and guide-wires, objects which the cardiologists are the most interested in, are the most active moving parts in the field of view. Thus it is relatively natural to try to combine spatial and temporal filtering methods in a synergistic manner. Indeed, this has been an active research topic in recent years $\mathrm{s}^{2,10,12,16-19,24}$. One problem emerging with spatial-temporal filtering is the detection of catheter (moving regions) in order to adaptively apply the distinct filtering techniques for static and moving regions within each frame. Several methods, such as template matching ${ }^{20}$, motion detection, or line structure detection ${ }^{16,21}$, etc. have been investigated and with good results.

The difference with previous methods and our method is the combination of techniques used. Temporal filtering gives very good results in noisy static regions. Due to the fact that temporal filtering may results in trailing artifacts of the catheter, also a catheter detection algorithm is implemented. In regions with motion spatial filtering is used. This combination results in an adaptive spatial-temporal filtering method for fluoroscopic image sequences.

In this context, we are proposing a multi-step adaptive spatial-temporal filtering technique for x-ray fluoroscopy angiography. First a normalized motion mask is generated. This mask describes the difference between two successive frames and the position of the catheter. Secondly each frame is spatially filtered using the specific motion mask to specify different types of filtering in each region. Third an IIR filter is then used to combine the spatially filtered image with the previous output image; the motion mask thus serves as a weighted input mask to determine how much spatial and temporal filtering should be applied.

The paper is organized as following: in the Method section, the general framework is explained in detail. In the Results section, processing results of our method on a real clinical fluoroscopy data set are presented. Finally, the Conclusions section presents our discussion and proposes possible future directions of research.

\section{METHOD}

Processing time varying image sequences to preserve or enhance the visibility of important parts of image sequences, such as catheter tips, anatomical structures and organs can be done adaptively by applying temporal filtering in regions without motion (static) and applying spatial filtering in regions with motion (dynamic). Our method focuses on combining spatial filtering with temporal filtering. The key problem in doing this is the detection of motion in these fluoroscopic image sequences. The frame rate is high enough to capture the motion of human organs, such as the beating heart, but the catheter occasionally jumps from one place to the other in subsequent frames. Exactly at the locations where the catheter jumps no temporal filtering should be applied. Therefore also a catheter detection algorithm is needed. An overview of our method is given in Fig. 1(a). In the following sections the individual processing steps will be described in more detail.

\subsection{Data}

Philips Medical Systems made three fluoroscopic image sequences available. All sequences consisted of 100 frames with each 960 squared pixels. The acquisition frame rate is typically between 15 and 30 frames per second.

\subsection{Motion mask}

The first order derivative in time, the amount of pixel intensity difference between frames, is normalized between 0 and 1. Where 0 denotes no difference between frames and 1 means maximum difference between frames. The higher the weight the less temporal filtering should be applied. The resulting motion mask describes the amount of difference between successive frames and thus the amount of temporal filtering to be applied between these frames. These weights are called the motion mask, because it gives a good estimation where the static regions and dynamic regions are located. 
All moving structures in the image sequences can be divided in two groups; moving human tissue and moving medical devices used during interventional procedures, mainly catheters and guide-wires. The medical devices, mainly line-like structures, need to be detected, because temporal filtering of such a device may result in trailing artifacts when the device moves between two successive frames. See Fig 1(c) for example trailing artifacts. Everywhere a moving line-like structure is detected the motion mask should be set to 1 , which will result in no temporal filtering.

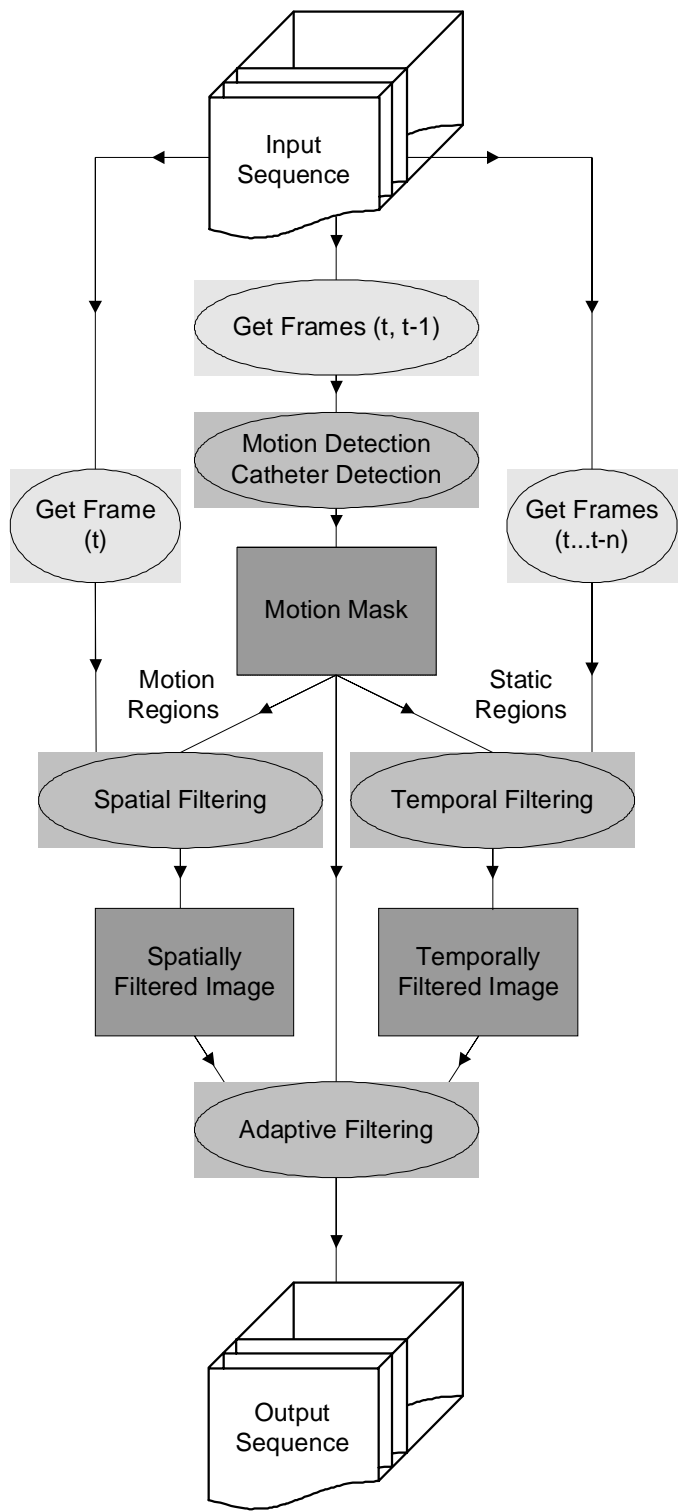

(a)

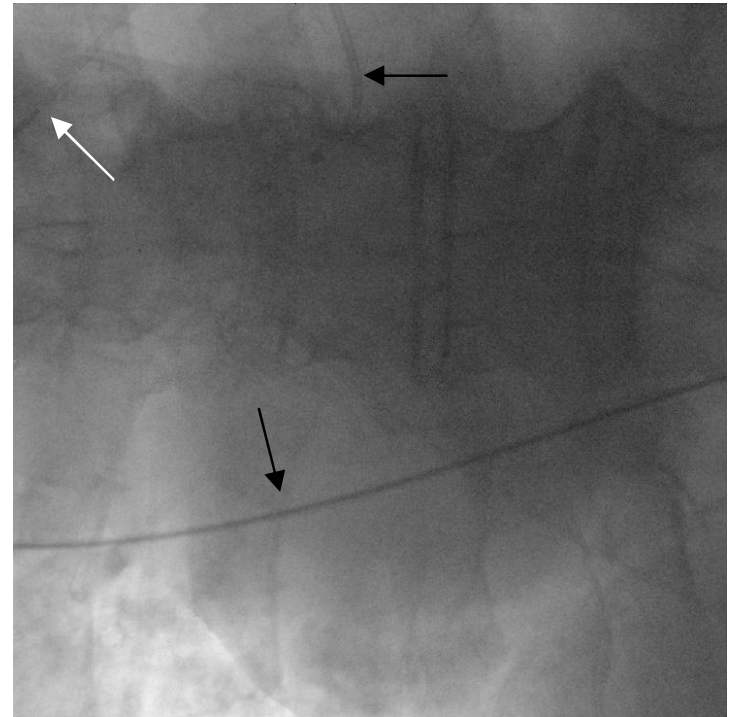

(b)

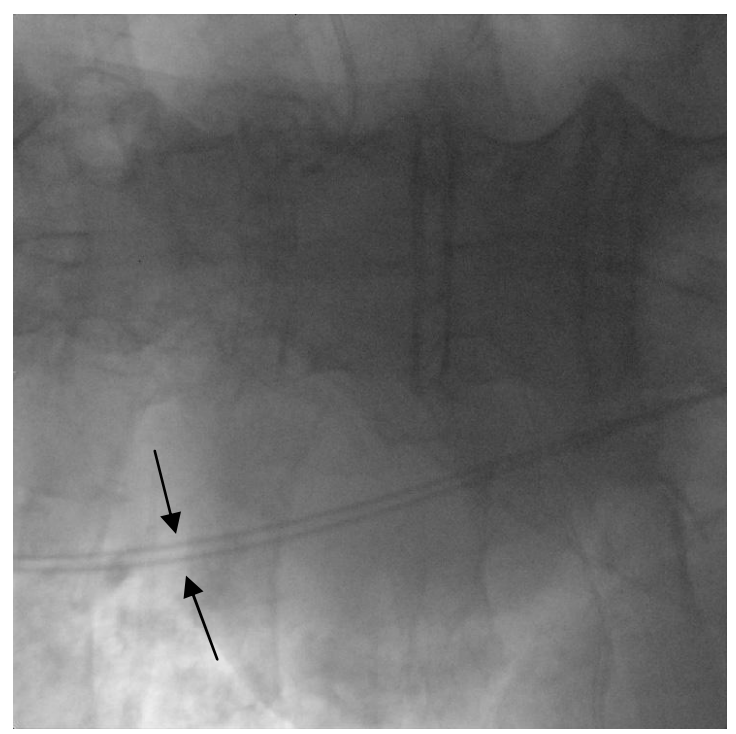

(c)

Figure 1: (a) Overview of processing steps and data flow for the adaptive filtering algorithm. (b) Typical x-ray fluoroscopic angiogram. Black arrows indicate catheters. The white catheter indicates the point where the guide-wire ends in the catheter. (c) Black arrows indicate trailing artifacts after temporal filtering: two catheters appear where only one catheter is present.

\subsection{Detecting moving line-like structures}

In order to set the motion mask to 1 where a line-like structure is moving, these structures first need to be detected. One of our methods to detect the line-like structures makes use of an eigenanalysis of the Hessian matrix [21], this is explained in the section 2.3.1 below. In section 2.3.2 an extension to moving line-like structures is explained. 


\subsubsection{Detecting line-like structures}

The Hessian Matrix is the second order Gaussian derivatives of the corresponding input image, and is defined as

$$
H=\left(\begin{array}{ll}
I_{x x} & I_{x y} \\
I_{y x} & I_{y y}
\end{array}\right)
$$

where $I_{x y}$ represents the convolution of the input image with the scaled second order Gaussian derivate

$$
I_{x y}=I * \frac{\partial^{2}}{\partial x \partial y} G_{2 D}(\vec{x}, \sigma)
$$

and $G_{2 D}(\vec{x}, \sigma)$ is the scaled Gaussian function

$$
G_{2 D}(\vec{x}, \sigma)=\frac{1}{2 \pi \sigma^{2}} e^{-\frac{|\vec{x}|^{2}}{2 \sigma^{2}}}
$$

An eigenanalysis of the Hessian matrix results in two eigenvectors and their corresponding eigenvalues $\lambda_{1}$ and $\lambda_{2}$, where $\lambda_{1}>\lambda_{2}$. On line-like structures, $\lambda_{1}$ has a large value and $\lambda_{2}$ a small value. This can be used to track line-like structures in an image. In Figure 2(B) the eigenvectors are scaled to the value of their corresponding eigenvectors and shown in double arrow plot glyphs.

The backtracking of line-like structures starts with looking for the largest $\lambda_{1}$. From that pixel a step in the direction of the eigenvector parallel to the line-like structure is made. After moving a certain amount of pixels $n$, three in the current implementation, a new pixel is found. If the pixel has an eigenvalue above a certain threshold we begin moving in the direction of the eigenvector parallel to the line-like structure in this pixel. Tracking the line-like structure stops when an eigenvalue is found beneath a different threshold or when we arrive at the border of the image.

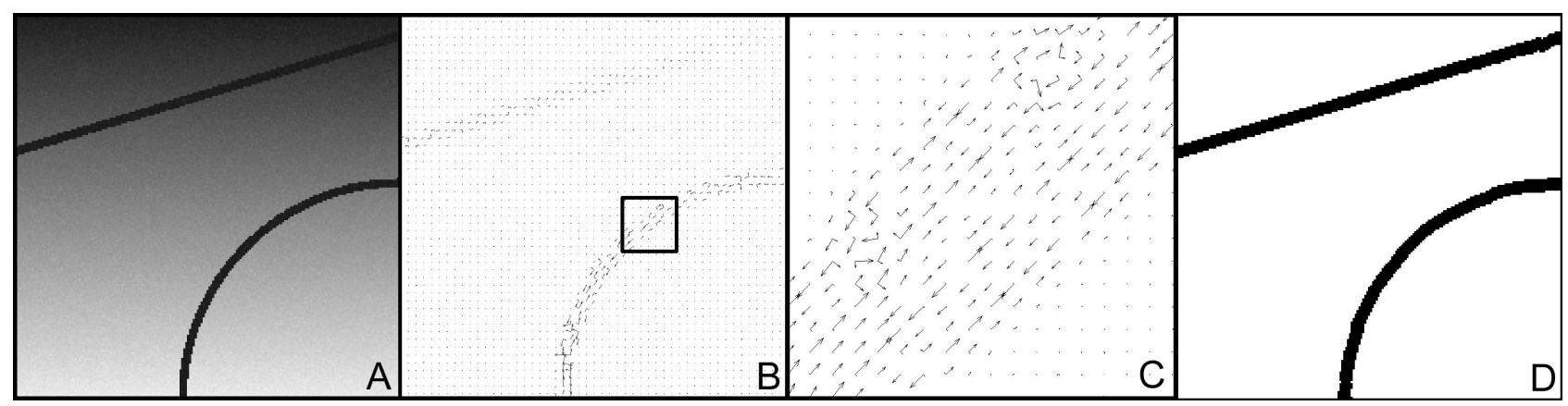

Figure 2: Example line tracking algorithm based on eigenvalues and eigenvectors of the Hessian matrix. (A) Test image with dimensions 200x200 pixels. The test image has a slowly varying background, a straight line, a curved line and randomly added noise. (B) Eigenvectors scaled to their eigenvalues shown in double arrow plot glyphs. (C) Enlargement from B: in the line-like structure the scaled eigenvectors are indeed larger than outside the line-like structures. (D) Result from the line detection algorithm.

\subsubsection{Detecting moving line-like structures}

To overcome trailing artifacts in the output sequence no temporal filtering should be applied to the moving catheter or guide-wire. Therefore all moving line-like structures need to be detected. The detection of line-like structures in single images, as explained in the previous section, can be applied to the images of the sequence, but also to the frame difference images. In the frame difference image mainly the moving pieces of the catheter are found and in a lesser extend the borders of anatomical structures and organs that are moving (e.g. heart, diaphragm and lung tissue). 
In the images from the sequence catheter pieces are detected and in a lesser extend borders of static anatomical structures and organs (e.g. bones). By only selecting line-like structures that are detected in both the input image and the frame difference image, the moving catheter can be selected. Objects in the frame difference images and input images may differ in size; the biggest object is selected to identify the whole catheter instead of only a short moving part. The following visual example demonstrates this process.

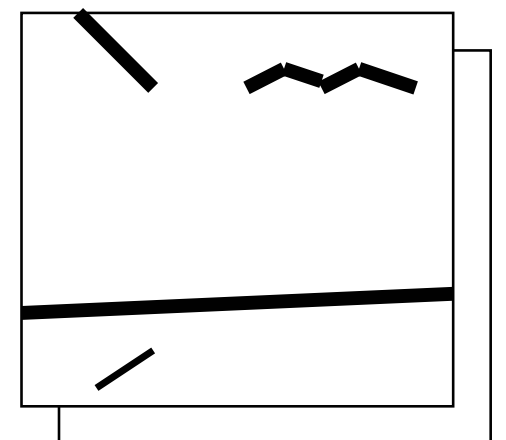

Line-like structures in input frames $i$ and $i-1$.

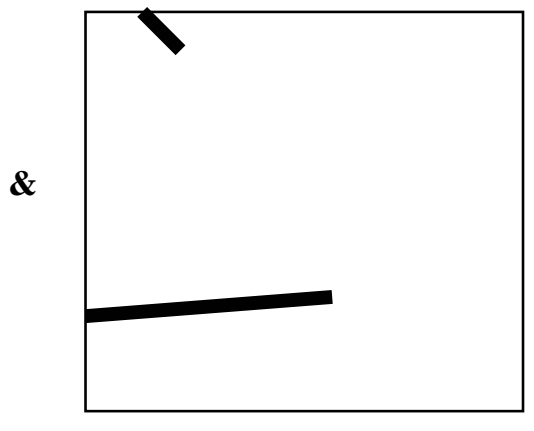

Detected moving line-like structures between frame $i$ and $i$ 1.

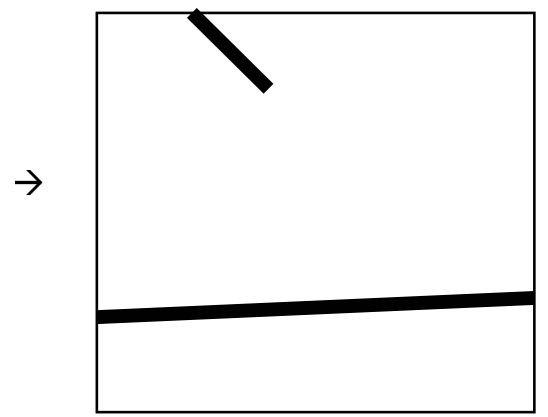

Moving catheter in frame $i$.

\subsection{Spatial filtering}

Many different spatial filtering techniques exist. The main goal of this application is to preserve the catheter or guidewire. The shape that needs to be preserved is a long elongated structure; therefore an adaptive filter is used. This filter uses a different rotated anisotropic Gaussian kernel for each pixel in the input image. The rotated anisotropic Gaussian kernel used to generate the kernels is defined as

$$
G\left(x, y, \sigma_{x}, \sigma_{y}, \theta\right)=\frac{1}{\sqrt{2 \pi \sigma_{x}^{2}}} \cdot e \frac{-(x \cos (\theta)+y \sin (\theta))^{2}}{2 \sigma_{x}^{2}} \cdot \frac{1}{\sqrt{2 \pi \sigma_{y}^{2}}} \cdot e \frac{-(-x \sin (\theta)+y \cos (\theta))^{2}}{2 \sigma_{y}^{2}}
$$

where $\sigma_{\mathrm{x}}$ and $\sigma_{\mathrm{y}}$ represent the width of the kernel in both directions and $\theta$ the angle of rotation over which the anisotropic kernel is rotated.

\subsection{Temporal filtering}

A first order IIR filter is used to temporally filter the image sequences. The output of the IIR filter is the weighted average of an input signal at time $t$, and the previous output signal:

$Y(t)=(1-c) * Y(t-1)+(c) * X(t)$

where $X$ is the spatially filtered input image, $Y$ the output image, and $c$ the motion mask with values between 0 and 1 . The motion mask is 1 for regions where the catheter is moving. In the remaining regions the pixels of the motion mask are between 0 and 1 dependent on the amount of difference between the pixels of successive frames.

\subsection{Adaptive filtering}

The final step in the process is adaptive filtering. A combination of the temporally filtered frame and the spatially filtered frame will result in the final output:

$Y(t)=(1-c) * T(t)+c * S(t)$

with $Y(t)$ adaptively filtered output frame at time $t, T$ temporally filtered frame, $S$ spatially filtered frame and $c$ motion mask with values between 0 and 1 , denoting no motion and motion respectively. 


\section{RESULTS}

\subsection{Moving catheter detection}

To evaluate the moving catheter detection algorithm 69 successive frames in a dataset were processed and the result was visually inspected. In 22 out of $69(32 \%)$ cases the whole catheter was detected. In only 7 out of 69 (10\%) cases no catheter or catheter parts were detected. In the remaining 40 cases (59\%) parts of the catheter were detected. In exactly one third of the cases the important tip part of the catheter was detected. See Table 1 for an overview of these results.

Table 1: Catheter detection results

\begin{tabular}{|l|l|}
\hline Detection result & Amount \\
\hline Full catheter detected & 22 \\
\hline With other structures detected & 14 \\
\hline No other structures detected & 8 \\
\hline Part of the catheter detected, and other structures detected & 25 \\
\hline Of which tip not detected & 24 \\
\hline Of which tip was detected & 1 \\
\hline Part of the catheter detected, and no other structures detected & 15 \\
\hline Of which tip not detected & 15 \\
\hline Of which tip was detected & 0 \\
\hline No parts of the catheter detected, but other structures & 6 \\
\hline Nothing detected & 1 \\
\hline Total & $\mathbf{6 9}$ \\
\hline
\end{tabular}

After the visual inspection of the results it was clear that the catheter algorithm performs better when the guide-wire is still in the catheter. This is caused by the fact that the catheter with guide-wire has a better contrast. In Figure 3(a) one frame is shown in which the catheter was fully detected without detecting other structures. The detection result is shown in white in Figure 3(b).

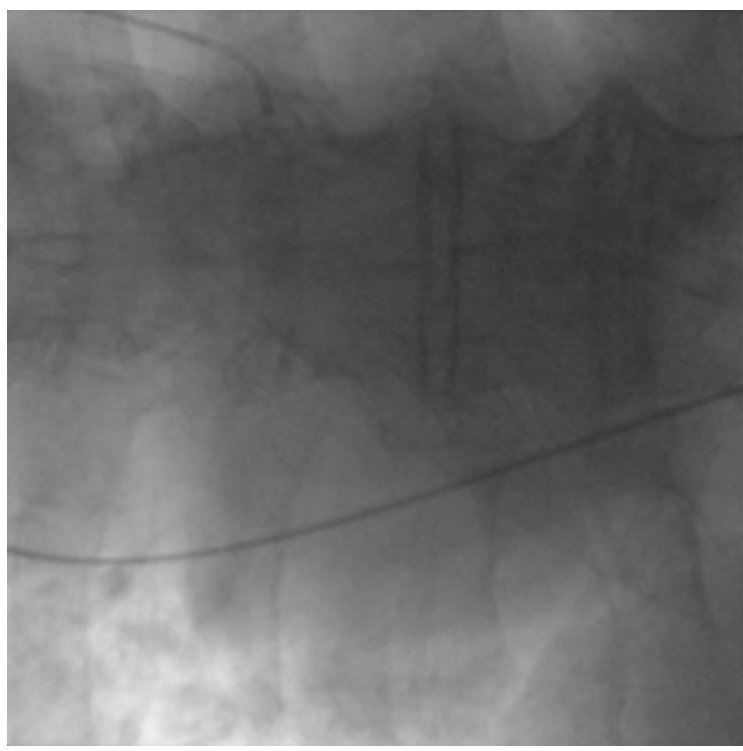

(a)

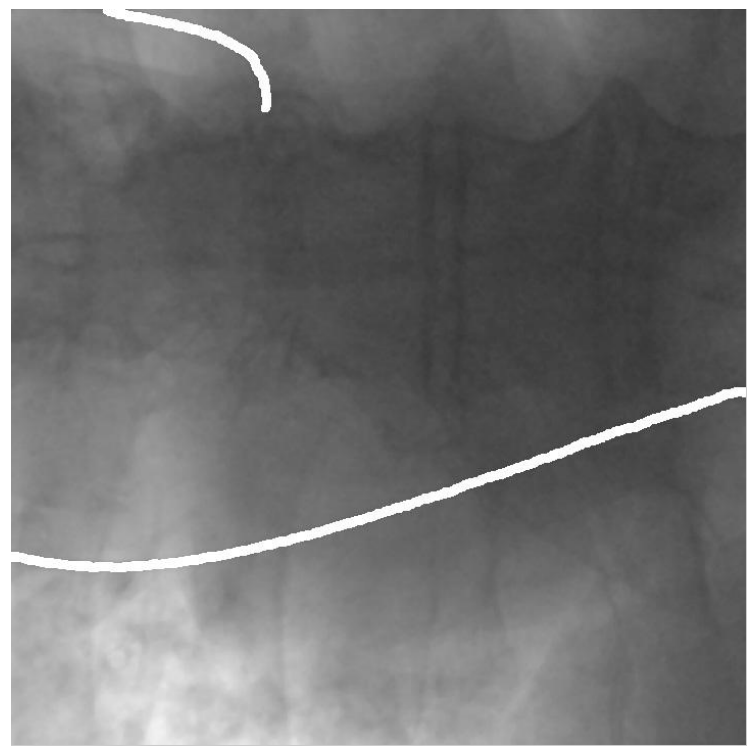

(b)

Figure 3: Catheter detection. (a) Input image with dimensions 960x960 pixels. (b) In white the detected catheter. The full catheter is detected correctly without detecting other structures. 


\subsection{Filtering}

After moving catheter detection and the generation of the motion mask the input image can be filtered. In Fig 4(a) a part from the input image is shown. After temporal filtering shown in Fig 4(b) the noise is reduced and the object, in this case a part of the spine, has an increased visibility.

In Fig 4(c) and Fig 4(d) the input image and corresponding adaptive spatial-temporal filtered image are shown. The adaptive spatial-temporal filtering shows a decrease in image noise and a decrease in visibility of catheter and anatomical structures. Trailing artifacts are not present in this frame. However if catheter detections fails to detected a part of the catheter, trailing artifacts can occur. In that case, temporal filtering is still limited due to the motion mask that has a relative high value at the position of the moving catheter due to the frame difference.

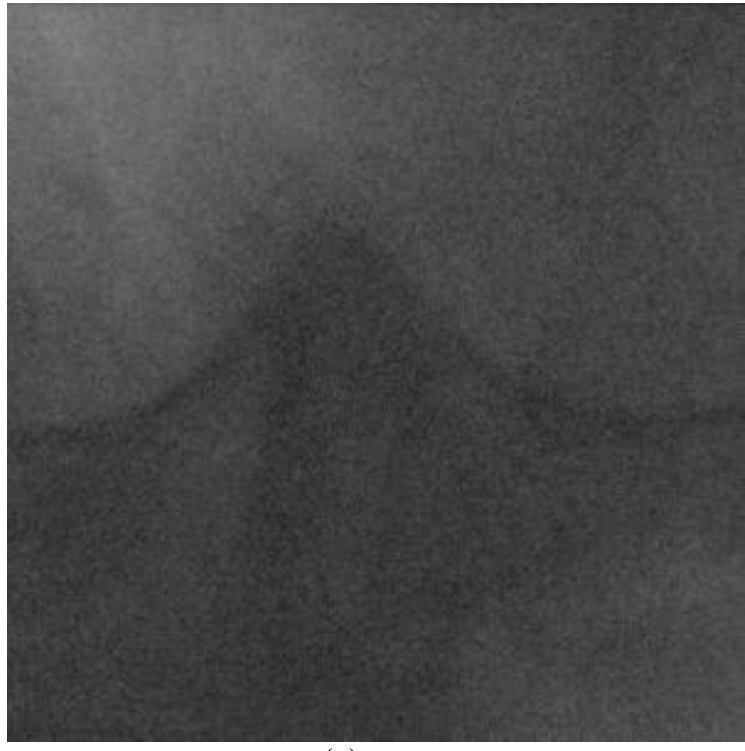

(a)

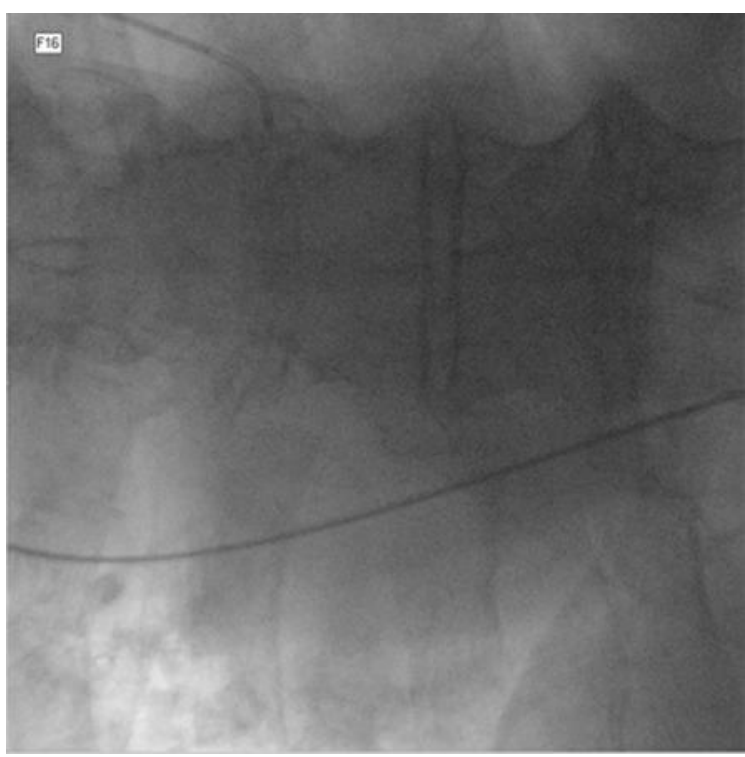

(c)

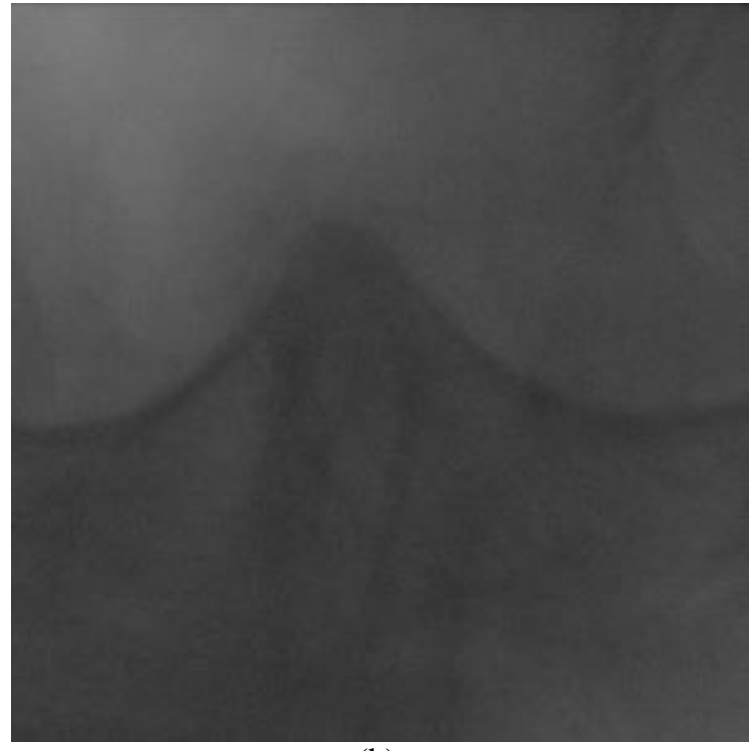

(b)

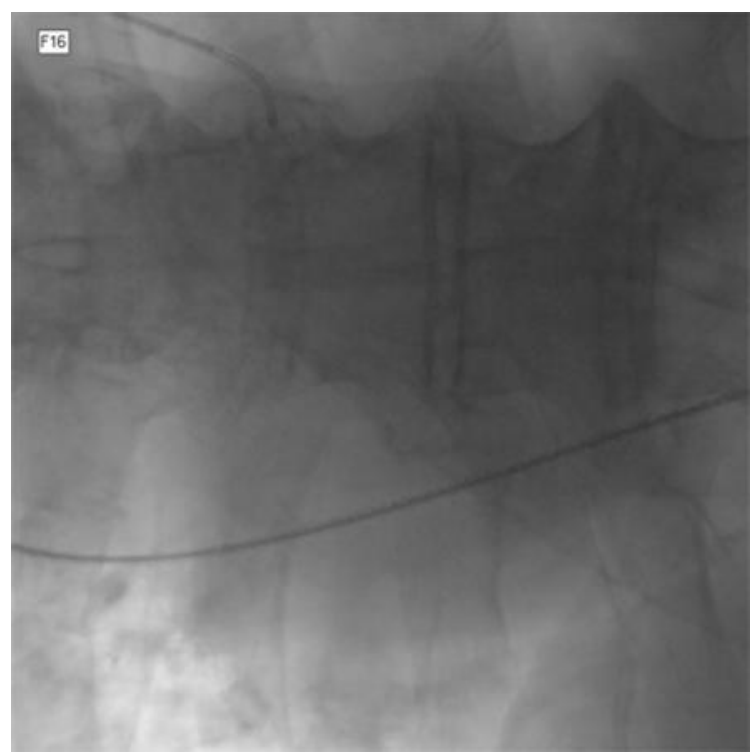

(d)

Figure 4: (a) Part of input frame, dimensions 300 squared pixels. (b) Temporal filtered image of the static region shown in (a). (c) Input frame, dimensions 960 squared pixels. (d) Adaptive filtered image of the input image (c). We acknowledge that it is difficult to appreciate the improvement in catheter motion by interpreting these two-dimensional static images. However, in the video playback the difference is dramatically clear. 


\section{CONCLUSION}

Existing techniques have difficulties filtering the image sequences; they suffer from blurring of catheters, especially at the tip, anatomical structures and moving organs such as the heart and lung, some also introduce trailing artifacts. We created a novel algorithm that can filter fluoroscopy X-ray image sequences acquired during endovascular interventions, reducing noise while minimizing spatial and motion blur. This technique preserves the sharpness of the tip of the catheter, which is important for navigation through vasculature during an interventional procedure.

The adaptive spatial-temporal filtering process for fluoroscopy x-ray image sequences was tested for visual quality. The results show that the visibility of anatomical structures and organs is improved due to the temporal filtering, while the moving catheter and guide-wire are preserved and spatially filtered. In addition, there are no trailing artifacts present in the processed image sequences when the catheter is detected successfully. In video playback the results can be clearly seen.

\section{FUTURE WORK}

The overall adaptive spatial-temporal filtering is promising for fluoroscopic image sequences. The individual steps, spatial filtering, temporal filtering and catheter detection, can be optimized, but the framework connecting these steps gives good results, namely noise reduction, preservation of motion and no introduction of trailing artifacts. Currently an evaluation in which radiologists evaluate the quality of this and other algorithms is set up. The use of a Steerable Dyadic Wavelet Transform [25] is still under investigation.

\section{ACKNOWLEDGEMENT}

The datasets were kindly provided by Philips Medical Systems, Best, the Netherlands. The Heffner Biomedical Imaging Laboratory, Columbia University; Philips Medical Systems; and Eindhoven University of Technology, The Netherlands supported this work.

The authors thank Allison Bell, Matthew Neimark, Yinpeng Jin and Mike Schacht for their feedback, suggestions and their help with preparing this paper.

\section{REFERENCES}

1. P. Xue and D. L. Wilson, "Detection of moving objects in pulsed-x-ray fluoroscopy," Journal of the Optical Society of America A: Optics and Image Science, and Vision, vol. 15, pp. 375-388, 1998.

2. D. L. Wilson, Y. Srinivas, F. J. Sanchez-Marin, and K. N. Jabri, "Quantitative image quality evaluation of an order-statistic filter in x-ray fluoroscopic imaging," in Medical Imaging 2000: Image Perception and Performance, Feb 16-Feb 17 2000, 3981, 43-48, San Diego, CA, USA, 2000.

3. $\quad$ T. Aach and D. Kunz, "Multiscale linear/median hybrid filters for noise reduction in low dose X-ray images," in Proceedings of the 1997 International Conference on Image Processing. Part 2 (of 3), Oct 26-29 1997, 2, 358-361, Santa Barbara, CA, USA, 1997.

4. $\quad$ K. N. Jabri and D. L. Wilson, "Quantitative image quality of spatially filtered X-ray fluoroscopy," Proceedings of SPIE - The International Society for Optical Engineering, Proceedings of the 1999 Medical Imaging - Image Perception and Performance, Feb 24-Feb 25 1999, vol. 3663, pp. 296-303, 1999.

5. $\quad$ K. N. Jabri and D. L. Wilson, "Quantitative image quality improvement in spatially filtered image sequences," Annual International Conference of the IEEE Engineering in Medicine and Biology, Proceedings, Proceedings of the 1999 IEEE Engineering in Medicine and Biology 21 st Annual Conference and the 1999 Fall Meeting of the Biomedical Engineering Society (1st Joint BMES / EMBS), Oct 13-Oct 16 1999, vol. 2, pp. 1142, 1999.

6. L. M. Fletcher, S. Rudin, and D. R. Bednarek, "Method for image equalization of ROI fluoroscopic images using mask localization, selection and subtraction," Computerized Medical Imaging and Graphics, vol. 20, pp. 89-103, 1996.

7. J. C. P. Heggie, "Usefulness of K-edge filters in automatic brightness controlled fluoroscopy and digital subtraction angiography," Australasian Physical \& Engineering Sciences in Medicine, vol. 15, pp. 9-14, 1992. 
8. K. N. Jabri and D. L. Wilson, "Detection improvement in spatially filtered x-ray fluoroscopy image sequences," Journal of the Optical Society of America A: Optics and Image Science, and Vision, vol. 16, pp. 742-749, 1999.

9. K. N. Jabri and D. L. Wilson, "Quantitative assessment of image quality enhancement due to unsharp-mask processing in x-ray fluoroscopy," Journal of the Optical Society of America A: Optics and Image Science, and Vision, vol. 19, pp. 1297-1307, 2002.

10. D. Kunz, K. Eck, H. Fillbrandt, and T. Aach, "A nonlinear multi-resolution gradient adaptive filter for medical images," in Medical Imaging 2003: Image Processing, Feb 17-20 2003, 5032 II, 732-742, San Diego, CA, United States, 2003.

11. P. Carre and C. Fernandez-Maloigne, "Use of the angle information in the wavelet transform maxima for image de-noising," Image and Vision Computing, vol. 18, pp. 1055-1065, 2000.

12. A. Singh, D. L. Wilson, and R. Aufrichtig, "Enhancement of x-ray fluoroscopy images," in Medical Imaging 1993: Image Processing, Feb 14-19 1993, 1898, 304-310, Newport Beach, CA, USA, 1993.

13. T. Aach and D. Kunz, "Bayesian motion estimation for temporally recursive noise reduction in X-ray fluoroscopy," Philips Journal of Research, vol. 51, pp. 231-251, 1998.

14. C. L. Chan, A. K. Katsaggelos, and A. V. Sahakian, "Enhancement of low-dosage cine-angiographic image sequences using a modified expectation maximization algorithm," in Visual Communications and Image Processing '92, Nov 18-20 1992, 1818, 290-298, Boston, MA, USA, 1993.

15. D. L. Wilson, K. N. Jabri, and R. Aufrichtig, "Perception of temporally filtered X-ray fluoroscopy images," IEEE Transactions on Medical Imaging, vol. 18, pp. 22-31, 1999.

16. R. Aufrichtig, D. Geiger, A. Singh, and D. L. Wilson, "Spatio-temporal x-ray fluoroscopy filtering using object detection," in Proceedings of the 1993 Conference on Computers in Cardiology, Sep 5-8 1993, 587-590, London, UK, 1993.

17. F. J. Sanchez-Marin, K. N. Jabri, Y. Srinivas, and D. L. Wilson, "Non-linear spatio-temporal filtering of X-ray fluoroscopy images," Annual International Conference of the IEEE Engineering in Medicine and Biology, Proceedings, Proceedings of the 1999 IEEE Engineering in Medicine and Biology 21 st Annual Conference and the 1999 Fall Meeting of the Biomedical Engineering Society (1st Joint BMES / EMBS), Oct 13-Oct 161999, vol. 2, pp. 1043, 1999.

18. K. Suzuki, S. Ikeda, and N. Imai, "Automatically controlled X-rays compensating filter system," Proceedings of SPIE - The International Society for Optical Engineering, Proceedings of the 1999 Medical Imaging Physics of Medical Imaging, Feb 21-Feb 23 1999, vol. 3659, pp. 645-652, 1999.

19. G. Wang, G. Schweiger, and M. W. Vannier, "Iterative algorithm for X-ray CT fluoroscopy," IEEE Transactions on Medical Imaging, vol. 17, pp. 853-856, 1998.

20. S. E. DeForest, "Improved fluoroscopic visibility of coronary stents by automatic pattern recognition," in Medical Imaging IV: Image Processing, Feb 6-8 1990, 1233, 464-472, Newport Beach, CA, USA, 1990.

21. S. A. M. Baert, M. A. Viergever, and W. J. Niessen, "Guide-wire tracking during endovascular interventions," IEEE Transactions on Medical Imaging, vol. 22, pp. 965-972, 2003.

22. P. Perona and J. Malik, "Scale space and edge detection using anisotropic diffusion," in IEEE Workshop on Computer Vision, 16-22, 1987.

23. P. Perona and J. Malik, "Scale-space and edge detection using anisotropic diffusion," IEEE Trans Pattern Anal Machine Intell, vol. 12, pp. 629-639, 1990.

24. R. Aufrichtig and D. L. Wilson, "X-Ray Fluoroscopy Spatio-Temporal Filtering with Object Detection", IEEE Transactions on Medical Imaging, Vol. 14, No. 4, pp. 733-746, 1995.

25. I. Koren, A.F. Laine, and F. Taylor, "Image Fusion Using Steerable Dyadic Wavelet Transform”, IEEE Int. Conf. Image. Process., Washington, D.C., Vol. 3, pp. 232-235, 1995. 